\title{
Necessity of heparin for maintaining peripheral venous catheters: A systematic review and meta-analysis
}

\author{
TAO YOU* , JIANLIANG JIANG* ${ }^{*}$ JIANCHANG CHEN, WEITING XU, LI XIANG and YANG JIAO \\ Department of Cardiology, The Second Affiliated Hospital of Soochow University, Suzhou, Jiangsu 215004, P.R. China
}

Received June 13, 2016; Accepted March 31, 2017

DOI: $10.3892 /$ etm.2017.4706

\begin{abstract}
Heparin has typically been used as a flushing or infusion solution for vascular lines in daily practice. However, several clinical trials have yielded controversial results about the benefits of heparin in maintaining peripheral venous catheters. The present meta-analysis was conducted to evaluate the efficacy of heparin on the patency profiles and complications in peripheral intravenous catheters. PubMed, Embase and Cochrane Central Register of Controlled Trials were searched up to February 2016 for randomized controlled trials comparing heparin with placebo in maintaining peripheral intravenous catheters. Additional studies were retrieved from the reference lists of identified articles. In total 32 eligible studies were included, from which the pooled standard mean difference (SMD), relative risk (RR) and corresponding 95\% confidence interval (CI) were calculated. The use of heparin as a continuous infusion significantly prolonged the duration of patency (SMD, 0.90; 95\% CI, 0.48-1.32; $\mathrm{P}<0.001)$, reduced rates of infusion failure (RR, 0.83; 95\% CI, 0.76-0.92; $\mathrm{P}<0.001)$ and occlusion ( RR, 0.82; 95\% CI, 0.69-0.98; $\mathrm{P}<0.05)$ in a peripheral intravenous catheter. However, there were no significant changes in the duration of patency and infusion failure when heparin was used intermittently as a flushing solution, although a significantly decreased risk of occlusion was observed in this setting (RR, 0.80; 95\% CI, 0.66-0.98; P<0.05). Furthermore, the risk of phlebitis was significantly decreased by both continuous infusion (RR, 0.66; 95\% CI, 0.58-0.75; P<0.01) and intermittent flushing (RR, 0.70; 95\% CI, 0.56-0.86; $\mathrm{P}<0.01$ ) of heparin in peripheral venous catheters. In conclusion, the use of heparin as continuous infusion in peripheral intravenous catheters improved the duration of patency, reduced infusion failure and phlebitis, whereas heparin as intermittent flushing
\end{abstract}

Correspondence to: Dr Yang Jiao or Dr Li Xiang, Department of Cardiology, The Second Affiliated Hospital of Soochow University, 1055 Sanxiang Road, Suzhou, Jiangsu 215004, P.R. China

E-mail: 15850070220@163.com

E-mail: 1229299337@qq.com

*Contributed equally

Key words: heparin, peripheral intravenous catheters, patency showed more benefits in ameliorating phlebitis rather than in patency profiles.

\section{Introduction}

Heparin has been widely used for the maintenance of intravascular catheters in patients requiring intravenous medications or arterial hemodynamic monitoring. The beneficial roles of heparin solution in ameliorating catheter occlusion, prolonging patency and reducing phlebitis have been investigated in a number of clinical studies $(1,2)$. As a classical anticoagulant-medicine, heparin is considered to prevent the formation of thrombus within the indwelling catheter while being used as a flushing or locking solution (3-6). However, other studies have reported no additional benefits from heparin compared to normal saline in promoting catheter patency (7-9). A previous systematic review and meta-analysis of randomized controlled trials (RCTs) indicated that intermittent flushing with heparin was not superior to normal saline in reducing catheter occlusion, whereas low dose infusion with heparin may improve the catheter patency and prevent phlebitis (4). Furthermore, two Cochrane systematic reviews were unable to provide conclusive evidence favoring the application of heparin solution over normal saline in the maintenance of central venous and arterial catheters $(10,11)$.

Previous studies on the use of heparin solution in peripheral venous catheters have shown controversial results. One Cochrane review that included both randomized and quasi-randomized trials reported significant heterogeneity among studies, which were not available for a meta-analysis (1). Another systematic review that focused on pediatric patients reported that continuous heparin infusion improved catheter patency, decreased infusion failures and lessened phlebitis. However, the same study showed no significant benefits of intermittent heparin flushing (3). A recent meta-analysis demonstrated similar beneficial roles of heparin solution in continuous infusion rather than intermittent flushing in adults (12).

Despite its wide use in daily practice, there are some concerns about the safety of heparin. For example, heparin has been shown to induce thrombocytopenia, and increasing bleeding risk and allergic reactions may occur following heparin infusion or flushes (13). Furthermore, a routine use of heparin for the most extensively placed peripheral venous catheters may unnecessarily increase the cost of health care 
when its cost-effectiveness remains unclear (14). As such, a number of RCTs with inconsistent or conflicting results have been published, prompting further debate on the use of heparin solution for peripheral venous catheter maintenance (15-17). Therefore, this updated systematic review and meta-analysis of RCTs was conducted to more precisely evaluate the role of heparin in maintaining peripheral venous catheters.

\section{Materials and methods}

Search strategy. A comprehensive literature search up to February 2016 was performed using PubMed (www. ncbi.nlm.nih.gov/pubmed), Embase (www.embase.com), Web of Science (www.isiknowledge.com) and Cochrane Central Register of Controlled Trials (www.cochranelibrary. com/about/central-landing-page.html) without language limitation. Manual searching was also used to identify eligible studies from key journals, major conference abstracts, original articles and reference lists. The following Medical Subject Headings and free text words were used: 'Heparin', 'placebo', 'control' and 'peripheral'. Furthermore, the search was restricted to human studies and RCTs.

Study selection. Two investigators independently assessed the articles, and any disagreements were resolved by consensus with a third reviewer. Studies were selected if they met the following criteria: i) RCTs, ii) conducted in subjects with peripheral venous catheters, iii) compared heparin added to intravenous fluid vs. no heparin added to the similar fluid and iv) measured catheter patency, occlusion, infusion failure or phlebitis. Furthermore, studies in which the subjects received systemic anticoagulation with heparin from any other access were excluded.

Data extraction. Abstracts and full texts of the studies included were retrieved by two reviewers independently and managed in Endnote X7 (Clarivate Analytics, Philadelphia, PA, USA). Data were extracted by two reviewers and further verified by a third reviewer, who then resolved the disagreements. The following data were extracted and stored in standardized forms: First author, publication year, country, study type, population and protocol, patient and catheter numbers, catheter material and size, heparin dosage and frequency of administration, outcomes and results.

Assessment of bias. The quality of the studies were evaluated using the Cochrane risk of bias tool (18), comprising of random sequence generation, allocation concealment, blinding, incomplete outcome data, selective reporting, and other bias. Any discrepancies were resolved by consensus with a third reviewer.

Outcomes. In the majority of the studies included, the primary outcomes of interest reported were catheter occlusion and patency duration. Secondary outcomes were infusion failure caused by any reasons, phlebitis and other major adverse events associated with the use of heparin. Phlebitis was determined when pain, induration, tenderness, erythema or palpable cord was recorded. Furthermore, major adverse events included heparin induced thrombocytopenia, allergy and major bleeding.

Data analysis. The effects of heparin solution from either continuous infusion or intermittent flushing studies were analyzed for subgroups. The total effects of dichotomous data using risk ratios (RRs) were pooled. Continuous outcomes were expressed as the mean \pm standard deviation (SD) and analyzed using standard mean differences (SMDs). Furthermore, the $\mathrm{I}^{2}$ test was conducted to assess the heterogeneity among the studies. Once significant heterogeneity was found $\left(\mathrm{I}^{2}>50 \%\right)$, the random effects models (Inversed-Variance) were used and a sensitivity analysis was performed to verify the consistency of the results. Alternatively, the fixed model (Mantel-Haenszel for dichotomous data and Inverse-Variance for continuous data) was selected for the analysis. The Egger's and Begg's tests, and funnel plots were used to assess the publication bias, and subgroup analysis and meta-regression were used to identify potential sources for heterogeneity between studies. Statistical analysis was performed with Stata (version 12; StataCorp LLC, College Station, TX, USA), using the metan, metareg and metainf commands amongst others. A two-sided $\mathrm{P} \leq 0.05$ was considered statistically significant.

\section{Results}

Study selection and characteristics. A standard workflow of searching and selection of eligible studies according to the PRISMA statement (19) is presented in Fig. 1. In total, 629 references from electronic databases were revealed by the literature search, of which, 293 were identified as duplicates due to the overlap of the databases. The remaining 336 citations were screened for eligibility, yielding 81 studies for full-text review. Further examination of the relevant reference lists of published reviews and meta-analyses identified 15 additional citations. Finally, 32 studies were found to comply with the predefined inclusion criteria. The characteristics of the final 32 studies included in the present review are displayed in Table I, among which, there are 21 reported results for intermittent flushing (7-9,14-17,20-33) and 11 evaluated heparin for continuous infusion (34-44).

Baseline characteristics. Among the 32 studies included in the present review, the scale ranged from 16 patients/16 catheters to 451 patients/1,257 catheters. In total, 10 studies reported multiple catheters per patient $(15-17,23,25,27,37-39,43)$, with the rest of the studies reported a single catheter per patient. The study populations included pediatric patients $(14,15,21-24$, $26-29,32,33,37-39,44)$, healthy volunteers and adults $(7-9,16,17$, 20,30,31,34-36,40-43), and the administered treatments in all studies primarily consisted of antibiotics and parenteral nutrition. Furthermore, the origin of countries reported included the USA $(7,8,14,20,26-28,30,32,33,35,43)$, the UK $(39,40)$, Germany (38), France (36), the Netherlands (22), Australia (44), Italy (17), Canada (25), Ireland (34), India (15,31) and China (16). In the infusion studies, the concentration of heparin varied between 0.1 and $2 \mathrm{U} / \mathrm{ml}$, whereas in the intermittent flush studies the concentration used varied between 2 and $100 \mathrm{U} / \mathrm{ml}$. Finally, the material of catheters included Teflon, 


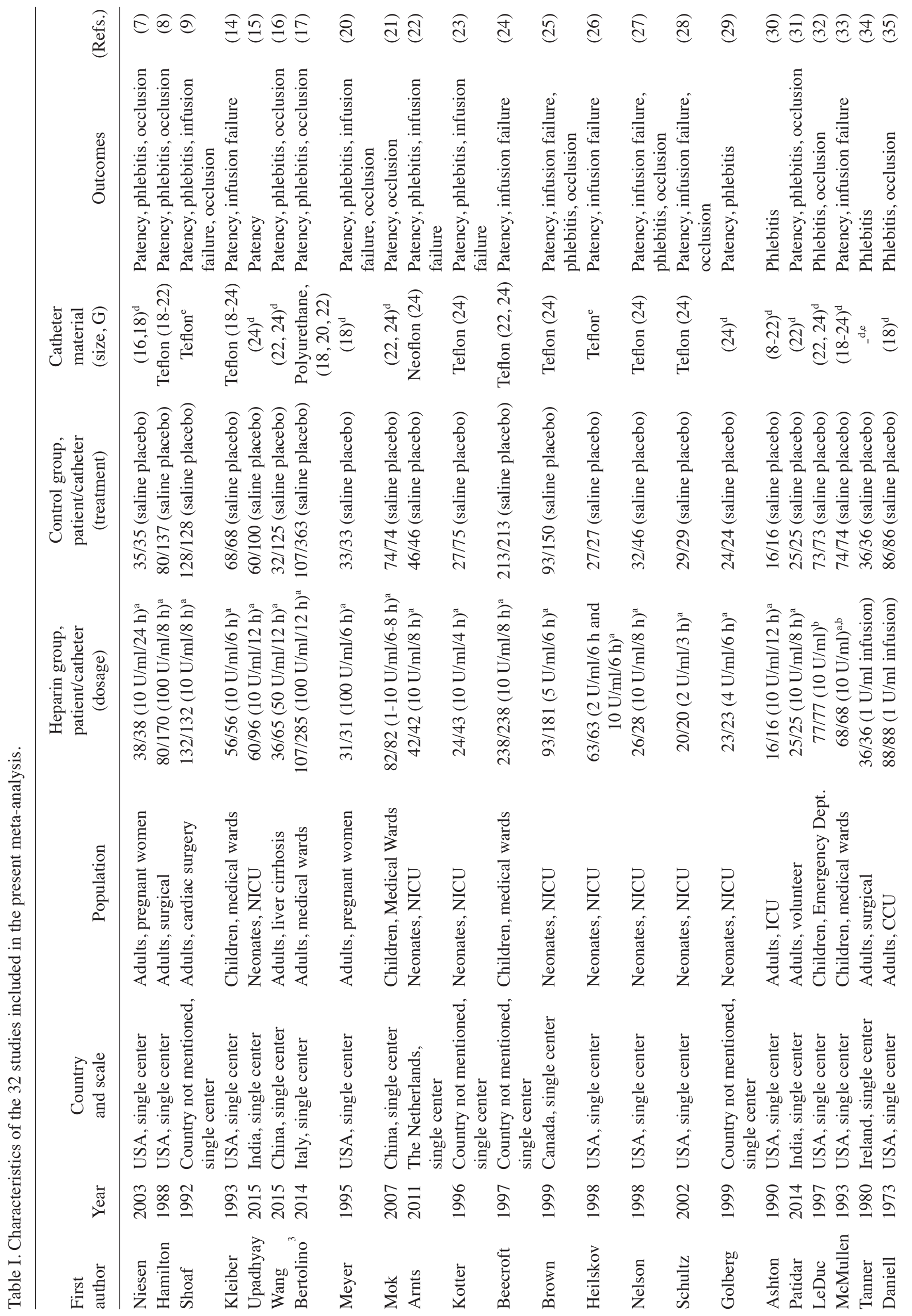




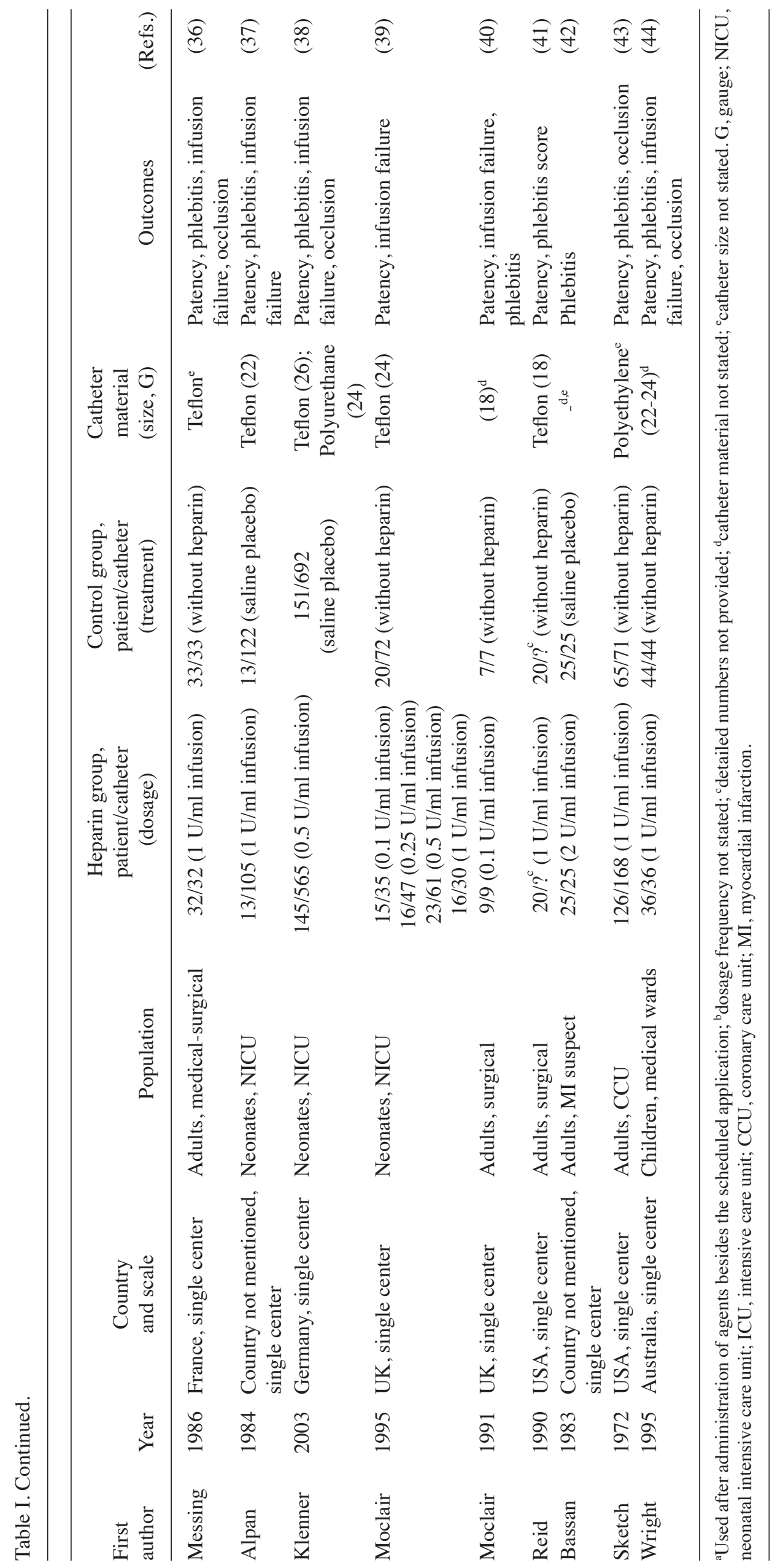




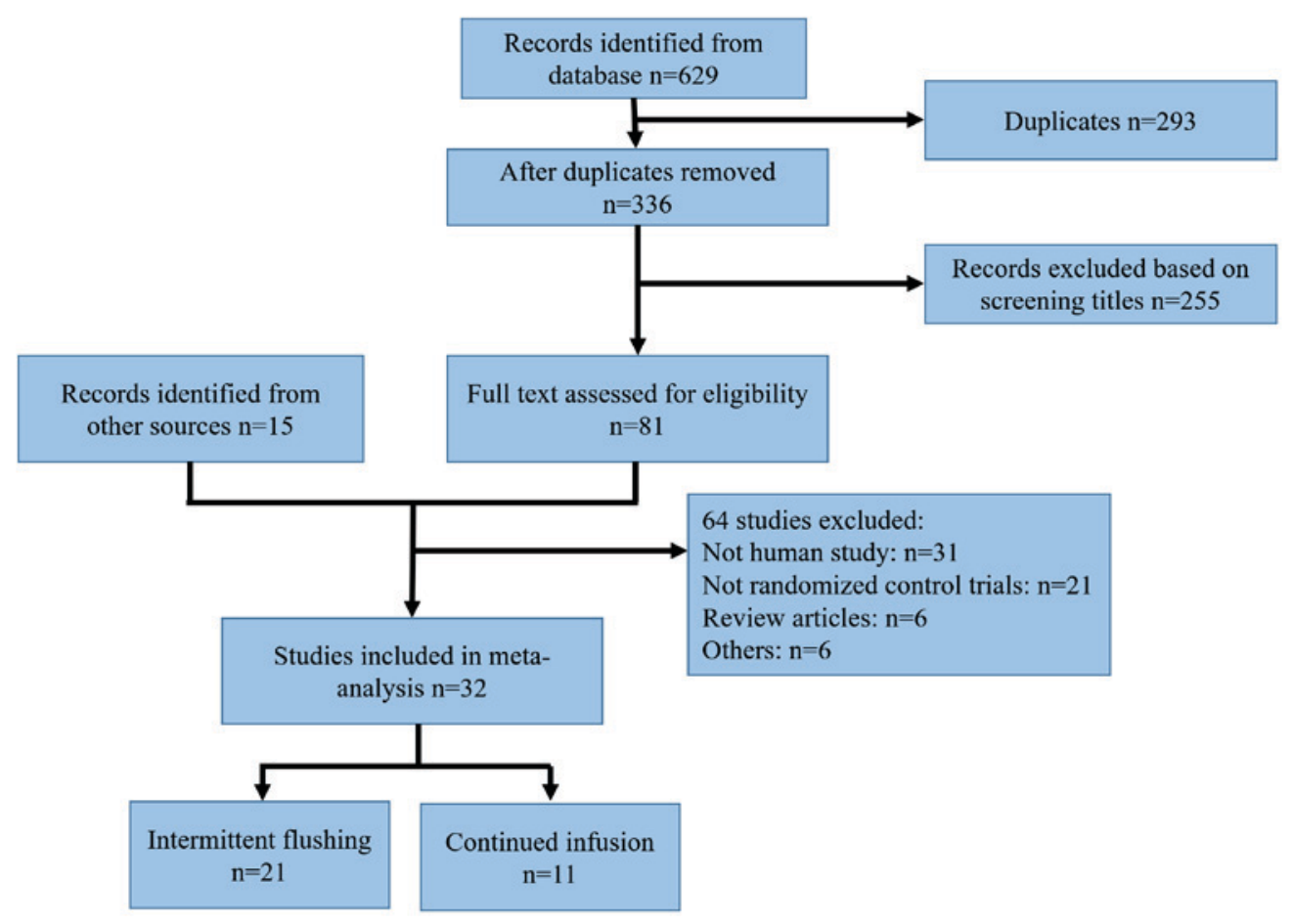

Figure 1. Workflow of study selection, in accordance with PRISMA.

polyurethane, Neoflon and polyethylene, and the size of catheters varied between 16 and 26 gauge.

Methodological quality. The majority of the studies demonstrated a low to unclear level of risk of bias, as shown in Fig. 2. In total, 10 studies did not properly address the methods for the generation of random sequence $(14,21,28,29,33,36$, $38,40-42)$. The majority of studies described an adequate method of allocation concealment, with 5 reported unclear results $(8,22,31,40,43)$. Furthermore, all studies reported well-defined methods of blinding except for 2 studies $(31,34)$. In terms of selective reporting and bias, however, the majority of studies did not provide a clear description. Although incomplete data were included in 5 studies, only small portions of subjects were removed following randomization. In addition, 7 studies did not provide drop-out details $(28,34,40-44)$.

\section{Meta-analysis}

Occlusion. Of the included studies, 21 reported the rates of occlusion per catheter, whereas 11 studies presented the occlusion rates per patient. The use of heparin in intermittent flushing lead to a $20 \%$ reduced risk in catheter occlusion [RR, 0.80; 95\% confidence interval (CI), 0.66-0.98; $\mathrm{P}<0.05$ ], and a similar reduction of the occlusion rate was exhibited in continuous heparin infusion studies (RR, 0.82; 95\% CI, $0.69-0.98 ; \mathrm{P}<0.05)$. In addition, the use of heparin also significantly decreased the overall risk of occlusion (RR, 0.81; 95\% CI, 0.71-0.93; $\mathrm{P}<0.01)$. Finally, no significant heterogeneity was observed among the studies (intermittent, $\mathrm{I}^{2}=26.9 \%$; continuous, $\mathrm{I}^{2}=5.5 \%$; overall, $\mathrm{I}^{2}=17.8 \%$; Fig. 3 ) and no publication bias was noticed among the studies as indicated by Egger's (continuous, $\mathrm{P}=0.962$; intermittent,
$\mathrm{P}=0.801$; overall, $\mathrm{P}=0.560$ ) and $\mathrm{Begg}$ 's (continuous, $\mathrm{P}=0.624$; intermittent, $\mathrm{P}=0.464$; overall, $\mathrm{P}=0.554$ ) tests.

Duration of catheter patency. The duration of catheter patency was reported in 22 studies, including 6 of continuous infusion and 16 of intermittent flushing. These studies described the duration in $\mathrm{h}$ (mean $\pm \mathrm{SD}$ ), which were summarized in Table I. In some studies, elective removal was issued for a quantity of catheters, which were separately recorded in the results. Other studies directly combined results from both elective and non-elective catheter removals. As shown in Fig. 4, continuous infusion of heparin solution in peripheral intravenous catheters significantly increased the duration of catheter patency (SMD, 0.90; 95\% CI, 0.48-1.32; P<0.001) whereas intermittent flushing with heparin did not display significant differences compared with placebo (SMD, 0.11; 95\% CI, -0.05-1.27; P=0.165; Fig. 4A). Furthermore, between-sample heterogeneities were significant in both groups (continuous, $\mathrm{I}^{2}=92.4 \%$; intermittent, $\mathrm{I}^{2}=72.0 \%$ ), therefore potential sources of these observations were sought. The continuous infusion studies demonstrated no publication bias, as assessed by Egger's $(\mathrm{P}=0.11)$ and Begg's $(\mathrm{P}=0.57)$ tests. However, in the intermittent flushing studies, the Egger's test showed significant publication bias $(\mathrm{P}<0.05)$ whereas the Begg's test did not display any significant difference. Notably, subgroup analysis revealed that both the duration of catheter patency (SMD, 0.30; 95\% CI, 0.17-0.42; $\mathrm{P}<0.01)$ and occlusion profile ( RR, 0.64; $95 \%$ CI, 0.48-0.86; $\mathrm{P}<0.01)$ were significantly improved when a higher concentration of heparin $(100 \mathrm{U} / \mathrm{ml})$ was used as an intermittent flushing solution.

Furthermore, a population-based subgroup analysis was conducted in the intermittent flushing studies and no significant 


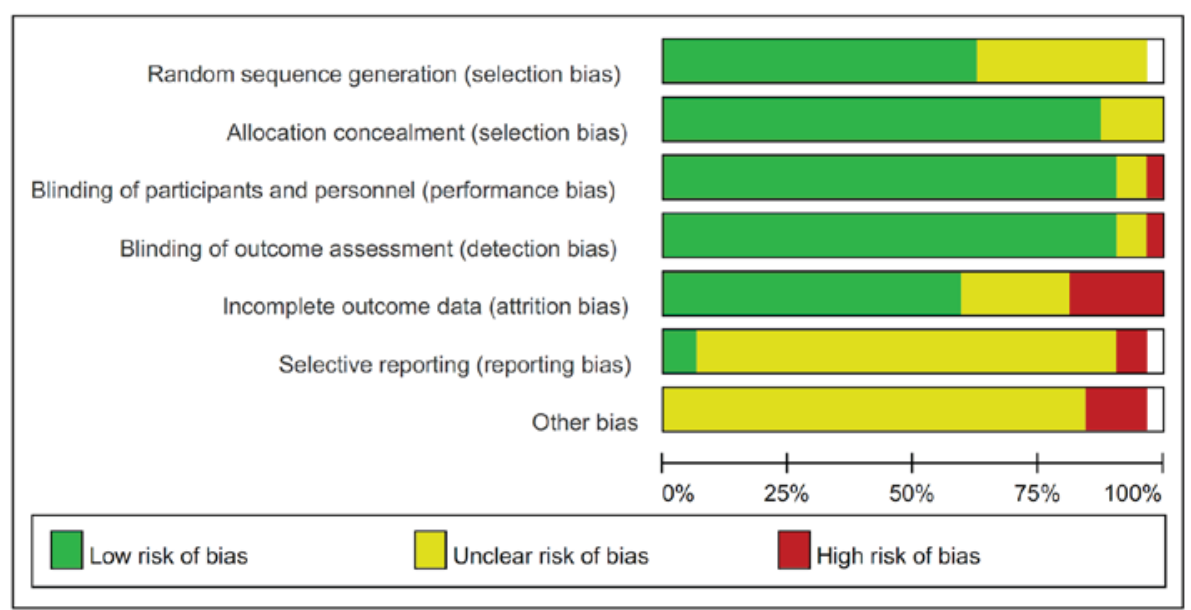

Figure 2. Assessment of methodological quality of the eligible studies using the Cochrane Collaboration's tool for risk of bias.

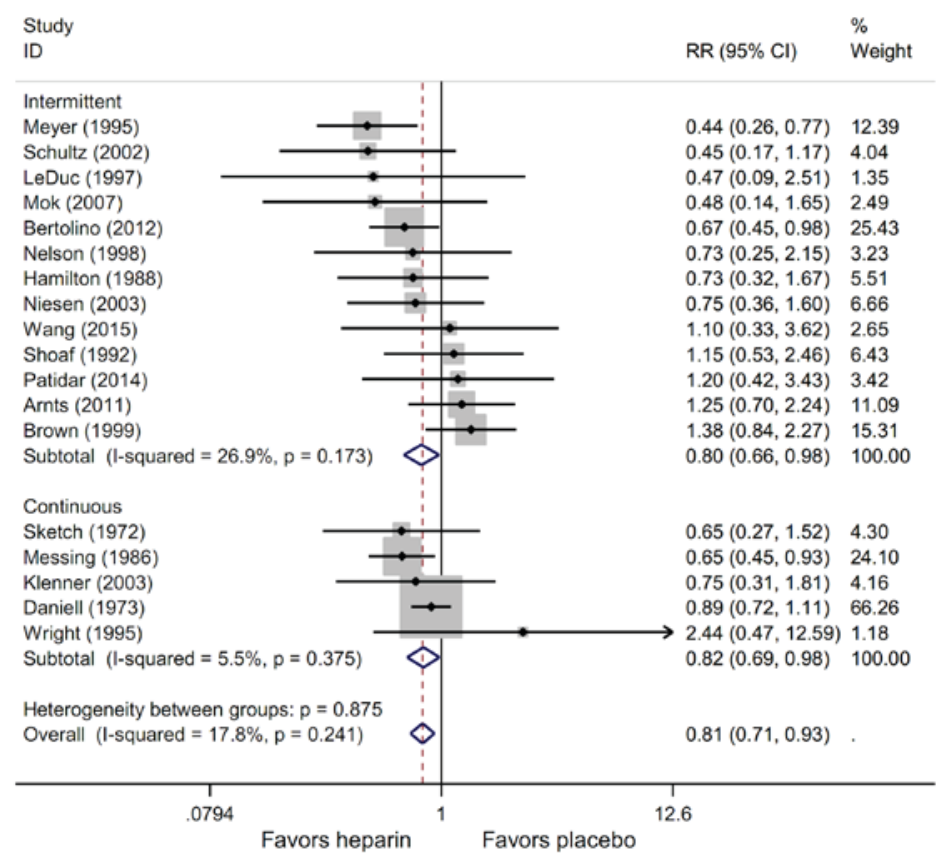

Figure 3. Meta-analysis for the RR of occlusion in peripheral intravenous catheters with or without heparin as intermittent flushing or continuous infusion. $\mathrm{RR}$, relative risk; CI, confidence intervals.

changes of catheter patency by heparin were detected in children (SMD, 0.10; 95\% CI, $-0.07-0.27 ; \mathrm{P}=0.25$ ) or adult population (SMD, 0.14; 95\% CI, -0.25-0.52; P=0.49). Homogeneity tests showed persistent between-study heterogeneity in both children $\left(\mathrm{I}^{2}=63.6 \%\right)$ and adults (continuous, $\mathrm{I}^{2}=85.3 \%$; Fig. 4B). Additionally, a univariate meta-regression test showed that the publication year, generation of random sequence, censor of elective catheter removal as well as catheter/patient ratio did not contribute significantly to the heterogeneity observed in the duration of patency.

Infusion failure. A total of 7 studies utilizing continuous infusion of heparin solution and 11 studies testing intermittent flushing with heparin presented this outcome as dichotomous data. A usage-based subgroup meta-analysis was performed for each group. Accordingly, only continuous application of heparin significantly reduced the risk of infusion failure in peripheral intravenous catheters (RR, 0.83; 95\% CI, 0.76-0.92; $\mathrm{P}<0.001$ ), whereas intermittent flushing with heparin failed to show a statistical difference (RR, 0.93; 95\% CI, 0.81-1.06; $\mathrm{P}=0.28$ ). Nevertheless, heparin demonstrated a significant overall effect on decreasing infusion failure in peripheral intravenous catheters (RR, 0.86; 95\% CI, 0.80-0.93; P<0.001). Further analysis showed no significant between-study heterogeneities (continuous, $\mathrm{I}^{2}=0 \%$; intermittent, $\mathrm{I}^{2}=49.5 \%$; overall, $\mathrm{I}^{2}=37.2 \%$; Fig. $5 \mathrm{~A}$ ). There was also no evidence of potential publication bias among the studies included as assessed by Begg's (continuous, $\mathrm{P}=0.453$; intermittent, $\mathrm{P}=0.938$; overall, $\mathrm{P}=0.678$ ) and Egger's tests (continuous, $\mathrm{P}=0.261$; intermittent, $\mathrm{P}=0.425$; overall, $\mathrm{P}=0.935$ ).

When heparin was used as intermittent flushing, no significant difference of infusion failure was noted in either children (RR, $-0.06 ; 95 \%$ CI, $-0.20-0.08 ; \mathrm{P}=0.40$ ) or adult (RR, -0.34 ; 

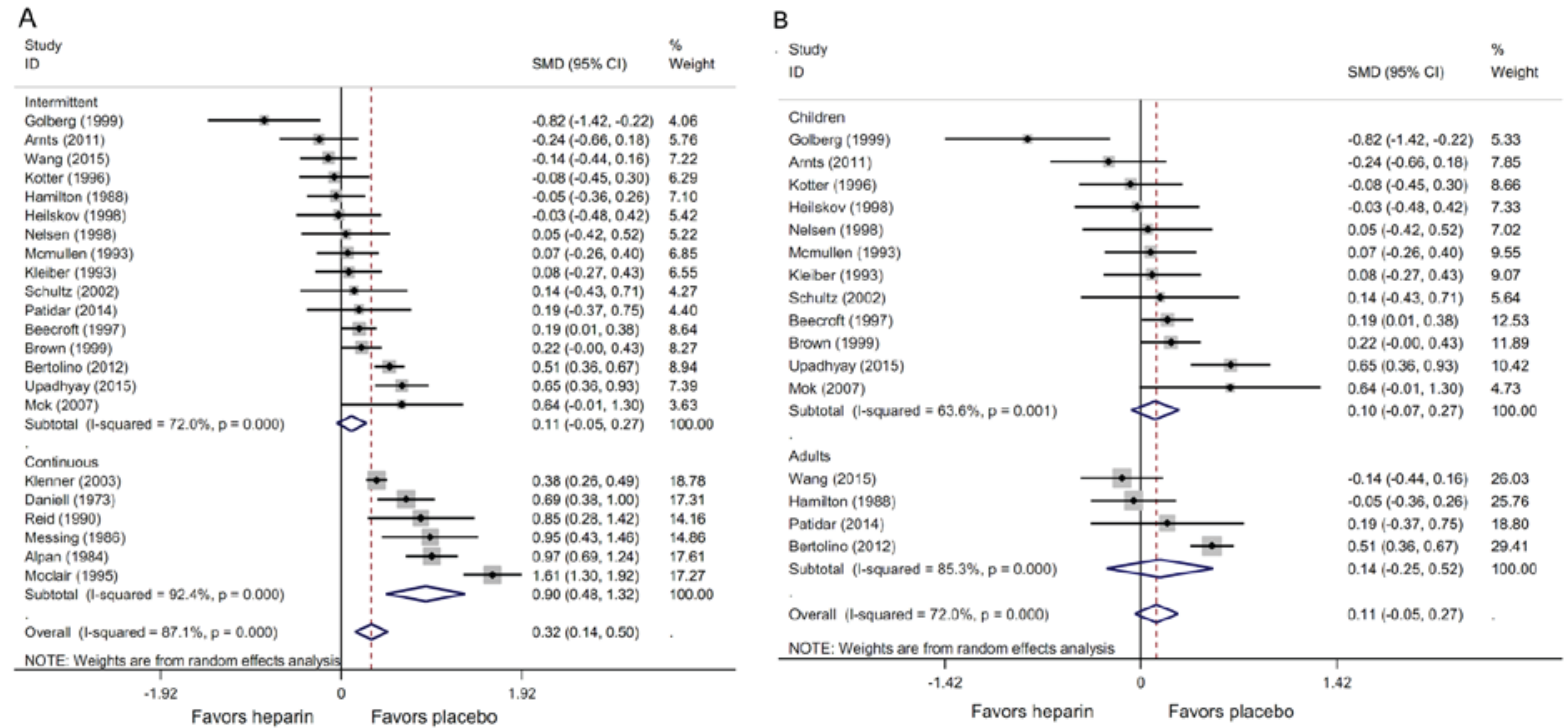

Figure 4. Quantitative analysis of the duration of patency. (A) Meta-analysis for the SMD of the duration of patency in peripheral intravenous catheters with or without heparin as intermittent flushing or continuous infusion. (B) Population-based subgroup analysis for the SMD of the duration of patency in peripheral intravenous catheters with heparin as intermittent flushing. SMD, standard mean differences; CI, confidence intervals.

A

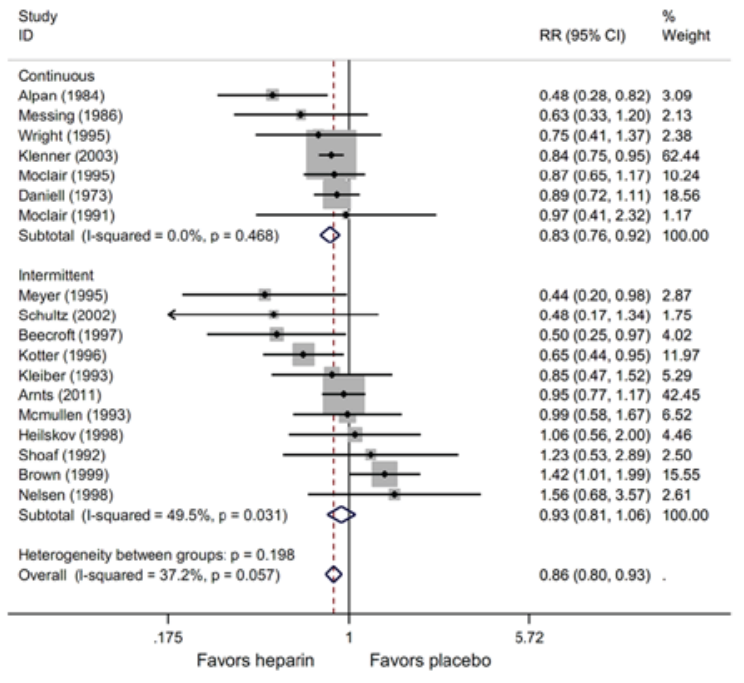

B

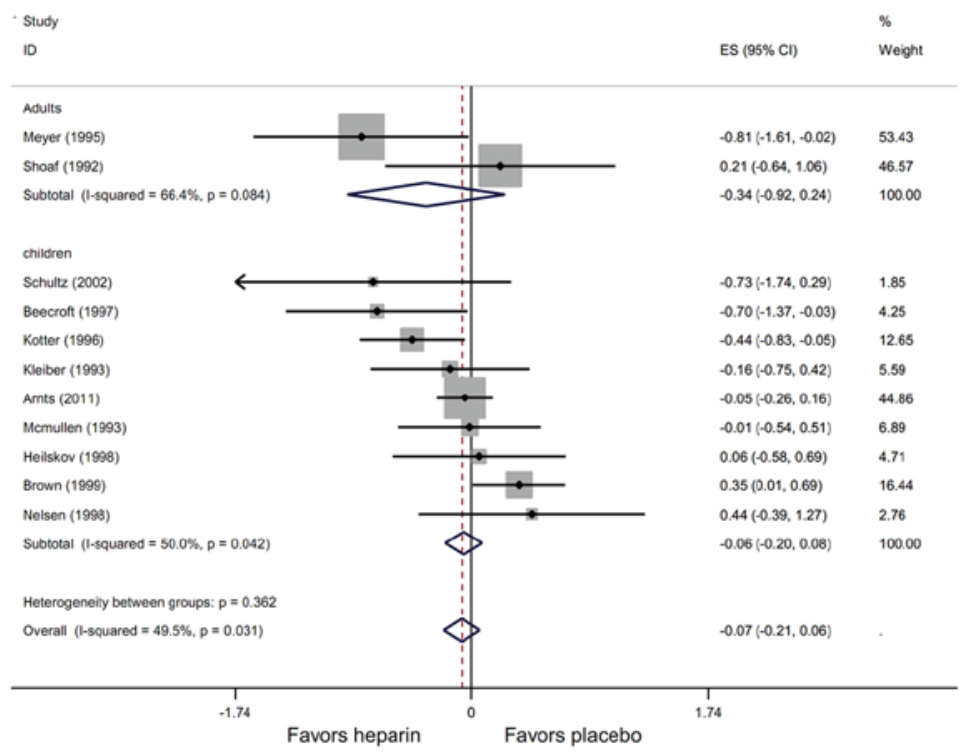

Figure 5. Quantitative analysis of infusion failure. (A) Meta-analysis for the rate of infusion failure in peripheral intravenous catheters with or without heparin as intermittent flushing or continuous infusion. (B) Population-based subgroup analysis for the rate of infusion failure in peripheral intravenous catheters with heparin as intermittent flushing. RR, relative risk; CI, confidence intervals; ES, effect size.

95\% CI, -0.92-0.24; $\mathrm{P}=0.26)$ populations, as indicated by population-based subgroup tests (Fig. 5B). Further analysis of meta-regression indicated no significant association between the years of publication, catheter/patient ratios, censoring for catheter removal, generation of random sequence and the heterogeneity noticed in infusion failure.

Phlebitis. The majority of included studies reported phlebitis as an outcome in binary format. According to the meta-analysis, the use of heparin significantly lowered the rates of phlebitis in both intermittent flushing (RR, 0.70; 95\% CI, 0.56-0.86; $\mathrm{P}<0.01)$ and continuous infusion $(\mathrm{RR}, 0.66$; 95\% CI, 0.58-0.75;
$\mathrm{P}<0.001)$ as compared with the placebo. The overall effect of heparin on reducing phlebitis was also statistically significant (RR, 0.67; 95\% CI, 0.60-0.75; P<0.001). Furthermore, the tests for homogeneity revealed a low level of heterogeneity among studies (continuous, $\mathrm{I}^{2}=21.1 \%$; intermittent, $\mathrm{I}^{2}=30.3 \%$; overall, $\mathrm{I}^{2}=21.9 \%$; Fig. 6 ), and no significant publication bias was indicated by Egger's (continuous, $\mathrm{P}=0.105$; intermittent, $\mathrm{P}=0.230$; overall, $\mathrm{P}=0.589$ ) and Begg's (continuous, $\mathrm{P}=0.095$; intermittent, $\mathrm{P}=0.807$; overall, $\mathrm{P}=0.527$ ) tests.

Major adverse events. One study reported adverse events, one of which reported 10 intracranial bleeding events, 


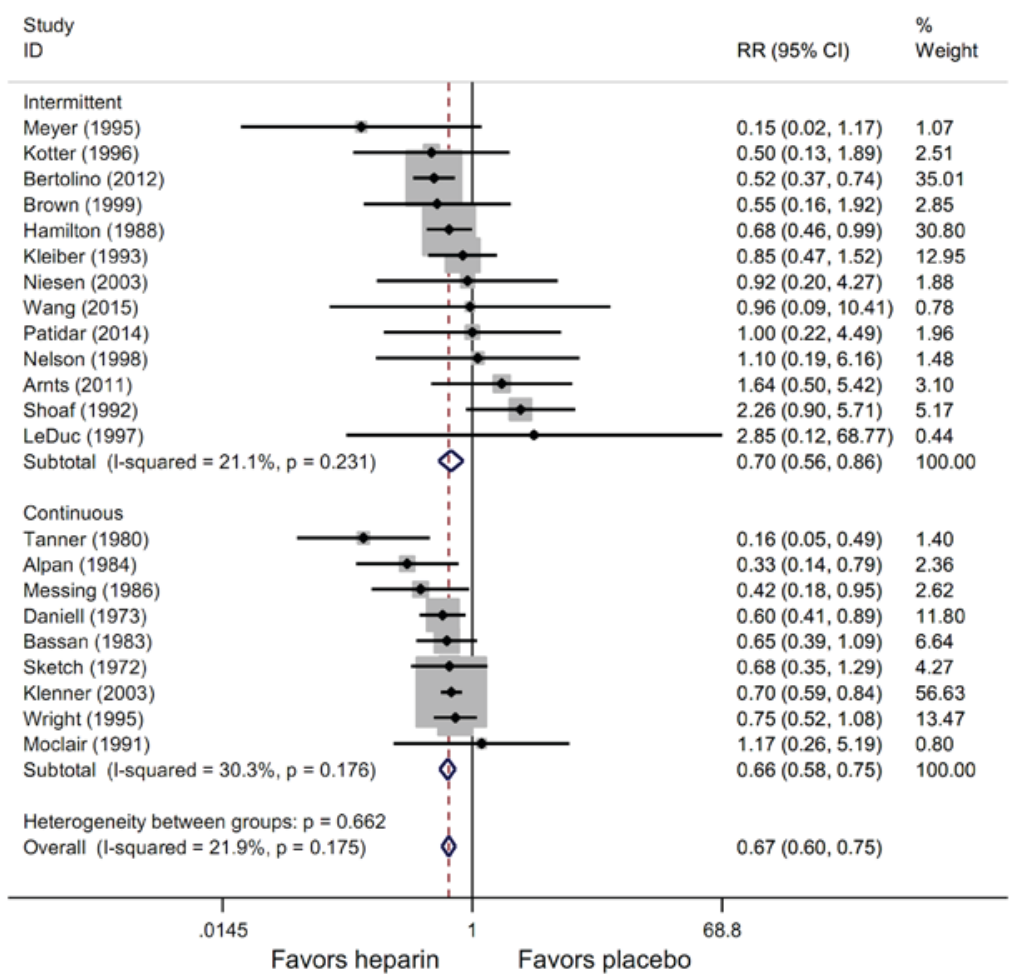

Figure 6. Meta-analysis for the relative risks of phlebitis in peripheral intravenous catheters with or without heparin as intermittent flushing or continuous infusion. RR, relative risk; CI, confidence intervals.

and 23 episodes of heparin-induced thrombocytopenia in neonates (38). Other studies only reported mild-bleedings, and no onset of sepsis or allergic reaction was noted $(17,25,38)$.

\section{Discussion}

Heparin has been utilized as an antithrombotic agent for maintaining catheters for four decades. Anticoagulation by heparin is predominantly mediated through antithrombin III in plasma (45). Due to rapid pharmacokinetics and a relatively lower cost, heparin is widely and routinely used in clinical practice. In some countries and medical facilities, flushing and locking catheters with heparin solutions are becoming standard procedures. However, a number of clinical trials studying heparin in catheter maintenance failed to find significance of its efficacy $(8,9,14,23,26)$. Therefore, several systematic reviews and meta-analyses are conducted but their results remain controversial $(3,4,11)$. Whereas many studies focus on central venous catheters, only a few have investigated the role of heparin in peripheral catheters. The present study performed an updated systematic review and meta-analysis of the efficacy of heparin solution used as both continuous infusion and intermittent flushing in peripheral venous catheters.

The results of the current meta-analysis demonstrated that the addition of heparin significantly reduced the rate of catheter occlusion, infusion failure and prolonged the duration of catheter patency in continuous infusion of a peripheral venous catheter. Conversely, heparin also showed a border effect on reducing occlusion and failed to display improvements in the duration of patency as well as infusion failure as an intermittent flushing solution. Nevertheless, a significant decrease was observed in the rate of phlebitis in both intermittent and continuous use of heparin.

The majority of infusion studies used low concentrations of heparin (1 U/ml) (34-39,41-44), whereas the majority of intermittent flushing studies used higher concentrations $(10-100 \mathrm{U} / \mathrm{ml})$. It is speculated that the difference of effects between these two distinctive modes of heparin usage may be due to discrepancies of concentrations. Notably, it was revealed that there were significant benefits in catheter patency when heparin was used for intermittent flushing at the highest concentration of $100 \mathrm{U} / \mathrm{ml}$. In addition, the pooled effect demonstrated that a higher frequency of flushing with heparin did not bring any further advantages in catheter patency. Additionally, one study of intermittent flushing with heparin showed no significant difference in catheter patency between concentrations of 1 and $10 \mathrm{U} / \mathrm{ml}$ (21). Conversely, an intergroup difference corresponding to concentration gradients of heparin was reported in one study using heparin as a continuous infusion (39).

In 1991, two meta-analyses including randomized and quasi-randomized studies were published, which reported that heparin solution was not superior to normal saline in maintaining patency of vascular catheters $(46,47)$. Another meta-analysis from 1998, which included only RCTs, demonstrated different results, in which heparin improved catheter patency during continuous infusion rather than intermittent flushing (4). However, in subgroup analysis, the authors revealed that a higher concentration of heparin exerted substantial benefits in catheter patency compared with the placebo. This result is in accordance with the present findings that a higher concentration of heparin was beneficial in intermittent flushing. More recently, two updated meta-analyses 
reported the efficacy of heparin on catheter patency in adults and children, respectively. In the first study, which focused on pediatric patients, heparin was shown to improve catheter patency while being continuously infused (3). In the second study, which focused on adult patients, inclusion of heparin in continuous infusion solutions resulted in improved patency outcomes and less phlebitis (12). Nonetheless, neither study reported significant benefits of heparin in intermittent application. Another systemic review by Shah and Shah (1) focused in neonates but did not conduct a meta-analysis due to high heterogeneity among the studies included, in which both semi-randomized and randomized trials were included.

Besides similar observations of phlebitis in the use of heparin as a continuous infusion, the present study demonstrated significant beneficial effects of heparin on preventing phlebitis as an intermittent flushing solution. Considering a similar duration of patency and occlusion profiles in the intermittent use of heparin compared with placebo was demonstrated in the present results, this improvement in phlebitis by heparin may be independent of catheter patency. Traditionally, phlebitis is considered to be caused by thrombosis and is usually called 'thrombophlebitis' (48). The observations of the present study that heparin showed additional benefits in preventing phlebitis besides anticoagulation may be explained by its anti-inflammatory role, which has been reported in sports injuries and inflammatory bowel disease $(49,50)$. Notwithstanding these benefits, administration of heparin may result in potential complications such as bleeding, thrombocytopenia and allergy (51-53). These drawbacks may discourage the clinical use of heparin at higher concentrations, although the morbidity of the complications has been low in previous observations $(7,13,16,51,53)$.

The meta-analysis of the present study showed improved outcomes of patency in the continuous infusion; however, the results must be interpreted with caution due to a series of limitations. First of all, high heterogeneity was observed in the analysis of duration of catheter patency. Furthermore, a subsequent meta-regression of the catheter/patient ratio, proper generation of randomization, year of publication and censoring for elective withdrawal failed to identify significant sources of heterogeneity. Additionally, since SMD was used as pooled effects for the continuous data as $h$, the discrepancies in the acquisition of outcomes among different studies may compromise the homogeneity of the results. Also, it is not possible to delineate the effects of the catheter material on the current observations in the present meta-analysis due to insufficient catheter information. The lack of sub-group studies with concentration gradients made it difficult to pool a concentration-dependent outcome. Additionally, there are other causes of bias in this review. For instance, a few studies included multiple groups that used other agents in combination with heparin $(36,42,43)$, and some other studies reported a considerable drop-out rate of the subjects $(9,35,42)$. Furthermore, one study reported the median rather than the mean and SD, and only effect size can be obtained from another $(39,44)$. It is noteworthy that one study with a large number of patients used cluster randomization instead of the classical randomization method (17). In addition, multi-center or large-scale RCTs were not identified for inclusion in the current meta-analysis. All of these factors may contribute to the heterogeneity and bias among the studies and affect the quality of the meta-analysis.

In conclusion, this updated systematic review and meta-analysis demonstrated that the use of heparin as a continuous infusion may significantly improve the patency of peripheral intravenous catheters, whereas intermittent flushing with heparin only reduces the rate of occlusion but shows no significant benefit on the duration of patency and infusion failure. Furthermore, the present findings suggest that both intermittent and continuous uses of heparin in peripheral intravenous catheters ameliorate phlebitis.

\section{Acknowledgements}

The authors are grateful to Dr Li Zhu (Soochow University; Suzhou, China) for his contributions to the search updates and manuscript preparation.

\section{References}

1. Shah PS and Shah VS: Continuous heparin infusion to prevent thrombosis and catheter occlusion in neonates with peripherally placed percutaneous central venous catheters. Cochrane Database Syst Rev: CD002772, 2008.

2. Unal S, Ekici F, Cetin II and Bilgin L: Heparin infusion to prevent umbilical venous catheter related thrombosis in neonates. Thromb Res 130: 725-728, 2012.

3. Kumar M, Vandermeer B, Bassler D and Mansoor N: Low-dose heparin use and the patency of peripheral IV catheters in children: A systematic review. Pediatrics 131: e864-e872, 2013.

4. Randolph AG, Cook DJ, Gonzales CA and Andrew M: Benefit of heparin in peripheral venous and arterial catheters: Systematic review and meta-analysis of randomised controlled trials. BMJ 316: 969-975, 1998.

5. Yau JW, Stafford AR, Liao P, Fredenburgh JC, Roberts R and Weitz JI: Mechanism of catheter thrombosis: Comparison of the antithrombotic activities of fondaparinux, enoxaparin, and heparin in vitro and in vivo. Blood 118: 6667-6674, 2011.

6. Daniell HW: Heparin prevention of catheter thrombosis. JAMA 216: 1200, 1971.

7. Niesen KM, Harris DY, Parkin LS and Henn LT: The effects of heparin versus normal saline for maintenance of peripheral intravenous locks in pregnant women. J Obstet Gynecol Neonatal Nurs 32: 503-508, 2003.

8. Hamilton RA, Plis JM, Clay C and Sylvan L: Heparin sodium versus $0.9 \%$ sodium chloride injection for maintaining patency of indwelling intermittent infusion devices. Clin Pharm 7: 439-443, 1988 .

9. Shoaf J and Oliver S: Efficacy of normal saline injection with and without heparin for maintaining intermittent intravenous site. Appl Nurs Res 5: 9-12, 1992.

10. Robertson-Malt S, Malt GN, Farquhar V and Greer W: Heparin versus normal saline for patency of arterial lines. Cochrane Database Syst Rev: CD007364, 2014.

11. Lopez-Briz E, Ruiz Garcia V, Cabello JB, Bort-Marti S, Carbonell Sanchis R and Burls A: Heparin versus $0.9 \%$ sodium chloride intermittent flushing for prevention of occlusion in central venous catheters in adults. Cochrane Database Syst Rev: CD008462, 2014.

12. Kumar M, Vandermeer B, Bassler D and Mansoor N: Low-dose heparin for prolonging the patency of peripheral intravenous catheters in adults - a systematic review and meta-analysis. Int J Med 2: 13-21, 2014.

13. Ahmed I, Majeed A and Powell R: Heparin induced thrombocytopenia: Diagnosis and management update. Postgrad Med J 83: 575-582, 2007.

14. Kleiber C, Hanrahan K, Fagan CL and Zittergruen MA: Heparin vs. saline for peripheral i.v. locks in children. Pediatr Nurs 19: 405-409, 376, 1993.

15. Upadhyay A, Verma KK, Lal P, Chawla D and Sreenivas V: Heparin for prolonging peripheral intravenous catheter use in neonates: A randomized controlled trial. J Perinatol 35: 274-277, 2015. 
16. Wang R, Zhang MG, Luo O, He L, Li JX, Tang YJ, Luo YL, Zhou M, Tang L, Zhang ZX, et al: Heparin saline versus normal saline for flushing and locking peripheral venous catheters in decompensated liver cirrhosis patients: A randomized controlled trial. Medicine (Baltimore) 94: e1292, 2015.

17. Bertolino G, Pitassi A, Tinelli C, Staniscia A, Guglielmana B, Scudeller L and Luigi Balduini C: Intermittent flushing with heparin versus saline for maintenance of peripheral intravenous catheters in a medical department: A pragmatic cluster-randomized controlled study. Worldviews Evid Based Nurs 9: 221-226, 2012.

18. Higgins JP, Altman DG, Gøtzsche PC, Jüni P, Moher D, Oxman AD, Savovic J, Schulz KF, Weeks L, Sterne JA, et al: The Cochrane Collaboration's tool for assessing risk of bias in randomised trials. BMJ 343: d5928, 2011

19. Moher D, Liberati A, Tetzlaff J and Altman DG; PRISMA Group: Preferred reporting items for systematic reviews and meta-analyses: The PRISMA statement. PLoS Med 6: e1000097, 2009.

20. Meyer BA, Little CJ, Thorp JA, Cohen GR and Yeast JD: Heparin versus normal saline as a peripheral line flush in maintenance of intermittent intravenous lines in obstetric patients. Obstet Gynecol 85: 433-436, 1995.

21. Mok E, Kwong TK and Chan MF: A randomized controlled trial for maintaining peripheral intravenous lock in children. Int J Nurs Pract 13: 33-45, 2007.

22. Arnts IJ, Heijnen JA, Wilbers HT, van der Wilt GJ, Groenewoud JM and Liem KD: Effectiveness of heparin solution versus normal saline in maintaining patency of intravenous locks in neonates: A double blind randomized controlled study. J Adv Nurs 67: 2677-2685, 2011.

23. Kotter RW: Heparin vs saline for intermittent intravenous device maintenance in neonates. Neonatal Netw 15: 43-47, 1996.

24. Beecroft PC, Bossert E, Chung K, Greene CV, Johnson LC, Jury D, Kent Y, O'Byrne C, Powell SL and Sperhac AM: Intravenous lock patency in children: dilute heparin versus saline. J Pediatr Pharm Pract 2: 211-223, 1997.

25. Brown K, Tay-Uyboco JS and McMillan DD: Heparin is not required for peripheral intravenous locks in neonates. Paediatr Child Health 4: 39-42, 1999.

26. Heilskov J, Kleiber C, Johnson K and Miller J: A randomized trial of heparin and saline for maintaining intravenous locks in neonates. J Soc Pediatr Nurs 3: 111-116, 1998.

27. Nelson TJ and Graves SM: $0.9 \%$ sodium chloride injection with and without heparin for maintaining peripheral indwelling intermittent-infusion devices in infants. Am J Health Syst Pharm 55: 570-573, 1998.

28. Schultz AA, Drew D and Hewitt H: Comparison of normal saline and heparinized saline for patency of IV locks in neonates. App Nurs Res 15: 28-34, 2002.

29. Golberg M, Sankaran R, Givelichan L and Sankaran K: Maintaining patency of peripheral intermittent infusion devices with heparinized saline and saline. Neonatal Intensive Care 12: 18-22, 1999.

30. Ashton J, Gibson V and Summers S: Effects of heparin versus saline solution on intermittent infusion device irrigation. Heart Lung 19: 608-612, 1990.

31. Patidar AB, Choudhary M, Bindu K and Midha V: Comparative efficacy of heparin saline and normal saline flush for maintaining patency of peripheral intravenous lines: A randomized control trial. IJHSR 4: 159-166, 2014.

32. LeDuc K: Efficacy of normal saline solution versus heparin solution for maintaining patency of peripheral intravenous catheters in children. J Emerg Nurs 23: 306-309, 1997.

33. McMullen A, Fioravanti ID, Pollack V, Rideout K and Sciera M: Heparinized saline or normal saline as a flush solution in intermittent intravenous lines in infants and children. MCN Am J Matern Child Nurs 18: 78-85, 1993.
34. Tanner WA, Delaney PV and Hennessy TP: The influence of heparin on intravenous infusions: A prospective study. Br J Surg 67: 311-312, 1980.

35. Daniell HW: Heparin in the prevention of infusion phlebitis. A double-blind controlled study. JAMA 226: 1317-1321, 1973.

36. Messing B, Leverve X, Rigaud D, Krummel Y, Botta D, Latarget J, Mudry $\mathrm{P}$ and Guignier M: Peripheral venous complications of a hyperosmolar (960 mOsm) nutritive mixture: The effect of heparin and hydrocortisone. A multicenter double-blinded random study in 98 patients. Clin Nutr 5: 57-61, 1986.

37. Alpan G, Eyal F, Springer C, Glick B, Goder K and Armon J: Heparinization of alimentation solutions administered through peripheral veins in premature infants: A controlled study. Pediatrics 74: 375-378, 1984.

38. Klenner AF, Fusch C, Rakow A, Kadow I, Beyersdorff E, Eichler P, Wander K, Lietz T and Greinacher A: Benefit and risk of heparin for maintaining peripheral venous catheters in neonates: A placebo-controlled trial. J Pediatr 143: 741-745, 2003.

39. Moclair A and Bates I: The efficacy of heparin in maintaining peripheral infusions in neonates. Eur J Pediatr 154: 567-570, 1995.

40. Moclair AE, Moselhi M, Benjamin IS and Hecker JF: Total parenteral nutrition via a peripheral vein: A comparison of heparinised and non-heparinised regimens. Int J Pharm Pract 1: 38-40, 1991.

41. Reid I, Keane FBV, Monson JRT and Tanner WA: Thrombophlebitis following peripherally administered parenteral nutrition-A randomized clinical study of the effect of infusion additives. Surg Res Commun 9: 69-77, 1990.

42. Bassan MM and Sheikh-Hamad D: Prevention of lidocaine-infusion phlebitis by heparin and hydrocortisone. Chest 84: 439-441, 1983.

43. Sketch MH, Cale M, Mohiuddin SM and Booth RW: Use of percutaneously inserted venous catheters in coronary care units. Chest 62: 684-689, 1972.

44. Wright A, Hecker J and McDonald G: Effects of low-dose heparin on failure of intravenous infusions in children. Heart Lung 24: 79-82, 1995.

45. Anderson AJ, Krasnow SH, Boyer MW, Raucheisen ML, Grant CE, Gasper OR, Hoffmann JK and Cohen MH: Hickman catheter clots: A common occurrence despite daily heparin flushing. Cancer Treat Rep 71: 651-653, 1987.

46. Goode CJ, Titler M, Rakel B, Ones DS, Kleiber C, Small S and Triolo PK: A meta-analysis of effects of heparin flush and saline flush: Quality and cost implications. Nurs Res 40: 324-330, 1991.

47. Peterson FY and Kirchhoff KT: Analysis of the research about heparinized versus nonheparinized intravascular lines. Heart Lung 20: 631-640, 1991

48. Brodin-Sartorius A, Martinez F and Legendre C: Treatment of superficial thrombophlebitis. N Engl J Med 364: 380-381, 2011

49. Tyrrell DJ, Horne AP, Holme KR, Preuss JM and Page CP: Heparin in inflammation: Potential therapeutic applications beyond anticoagulation. Adv Pharmacol 46: 151-208, 1999.

50. Folwaczny C, Wiebecke B and Loeschke K: Unfractioned heparin in the therapy of patients with highly active inflammatory bowel disease. Am J Gastroenterol 94: 1551-1555, 1999.

51. Pföhler C, Müller CS, Pindur G, Eichler H, Schäfers HJ, Grundmann U and Tilgen W: Delayed-type heparin allergy: Diagnostic procedures and treatment alternatives-a case series including 15 patients. World Allergy Organ J 1: 194-199, 2008.

52. Karaaslan H, Peyronnet P, Benevent D, Lagarde C, Rince M and Leroux-Robert C: Risk of heparin lock-related bleeding when using indwelling venous catheter in haemodialysis. Nephrol Dial Transplant 16: 2072-2074, 2001.

53. Mohamed M, Hayes R and Mosetlhi T: A man with acute venous thromboembolism and thrombocytopenia. Heparin induced thrombocytopenia. BMJ 348: g1164, 2014. 